\title{
Recovery of p-modes in the combined 2004-2005 MOST ${ }^{\star}$ observations of Procyon (Research Note)
}

\author{
S. V. Marchenko \\ Department of Physics and Astronomy, Western Kentucky University, 1906 College Heights Blvd., 11077, \\ Bowling Green, KY 42101-1077, USA \\ e-mail: sergey.vm@gmail.com
}

Received 6 August 2007 / Accepted 31 December 2007

\begin{abstract}
Aims. Procyon A, a bright F5 IV-V Sun-like star, is justifiably regarded as a prime asteroseismological target. This star was repeatedly observed by MOST, a specialized microsatellite providing long-term, non-interrupted broadband photometry of bright targets. So far, the widely anticipated p-modes eluded direct photometric detection, though numerous independent approaches hinted for the presence of signals in the $f \sim 0.5-1.5 \mathrm{mHz}$ range.

Methods. Implementation of an alternative approach in data processing, as well as combination of the MOST data from 2004 and 2005 (264 189 measurements in total) helps to reduce the instrumental noise affecting previous reductions, bringing the $3 \sigma$ detection limit down to $\sim 5.5$ part-per-million in the $f=0.8-1.2 \mathrm{mHz}$ range.

Results. This enabled us to cross-identifiy 16 p-mode frequencies (though not their degrees) which were previously detected via high-precision radial velocity measurements, and provides an estimate of the large spacing, $\delta v=0.0540 \mathrm{mHz}$ at $f \sim 1 \mathrm{mHz}$. The relatively low average amplitude of the detected modes, $a=5.8 \pm 0.6 \mathrm{ppm}$, closely matches the amplitudes inferred from the groundbased spectroscopy and upper limits projected from WIRE photometry. This also explains why such low-amplitude signals eluded the direct-detection approach which exclusively relied on the MOST 2004 (or 2005) data processed by a standard pipeline.
\end{abstract}

Key words. stars: oscillations - stars: individual: Procyon A - techniques: photometric - methods: data analysis

\section{Introduction}

Procyon A (F5 IV-V) has served as a prime target in many asteroseismological campaigns (see the reviews by Bedding \& Kjeldsen 2006, 2007, and references therein). While highprecision measurements of radial velocities revealed the presence of variability caused by solar-like oscillations (Martić et al. 1999) and provided a preliminary identification of p-mode frequencies (Eggenberger et al. 2004; Martić et al. 2004; Leccia et al. 2007), the photometric studies met either limited success (Bruntt et al. 2005) or outright null-detection (Matthews et al. 2004; Guenther et al. 2007). The null-detection result was hotly debated ever since (Bedding et al. 2005; Régulo \& Roca Cortés 2005). Attempting to resolve the controversy, we applied an alternative data-processing approach to the abundant photometric data acquired by MOST in 2004-2005.

\section{Observations and data processing}

The MOST satellite (Walker et al. 2003) is a $15 \mathrm{~cm}$ telescope acquiring high-precision, non-stop CCD photometric data on bright objects through a broad-band, $350-700 \mathrm{~nm}$, optical filter. MOST observed Procyon A in 2004 (32-day, non-stop observations, 2315240.9 s exposures: see the results discussed in

\footnotetext{
* MOST is a Canadian Space Agency mission, operated jointly by Dynacon, Inc., and the Universities of Toronto and British Columbia, with assistance from the University of Vienna.
}

Matthews et al. 2004), 2005 (taken over 17 sequential days with the general setup of the experiment following the 2004 campaign: Guenther et al. 2007) and 2007 (data are being processed: Matthews 2007, priv. comm.).

We took the publicly available raw data from 2004 and 2005 and processed them using an alternative algorithm (see the description in Aerts et al. 2006). While generally conforming to the approach of the standard processing pipeline applied to the 2004-2005 data, the alternative algorithm treats the highly variable stray light, the main source of instrumental noise, in a more flexible manner. In addition to the iterative subtraction of scattered light, the algorithm accounted for less substantial sources of instrumental noise, such as sensitivity drift caused by slow variations of the CCD temperature. The alternative approach resulted in a lower point-to-point scatter, $s=440$ part-per-million (ppm), vs. $s=500$ ppm (Matthews et al. 2004) (cf. the upper section of Fig. 1). This should be compared to the projected level of the Poisson-dominated instrumental noise $s_{\text {in }}=260 \mathrm{ppm}$. The alternative approach also reduces the average (noise-dominated) amplitudes and scatter in the frequency spectrum: e.g., for $f=$ $1.05-1.25 \mathrm{mHz}$ one obtains $\bar{a}(f)=2.8 \pm 1.7 \mathrm{ppm}$ in the older reduction vs. $\bar{a}(f)=2.6 \pm 1.4$ for the new algorithm (Fig. 1).

The modified approach, when efficiently combining the two available data streams (SDS1 and SDS2: see the definition in Aerts et al. 2006), proves to be even more effective with the 2005 data where the better planning of experiment allowed more straightforward suppression of the stray-light component, 

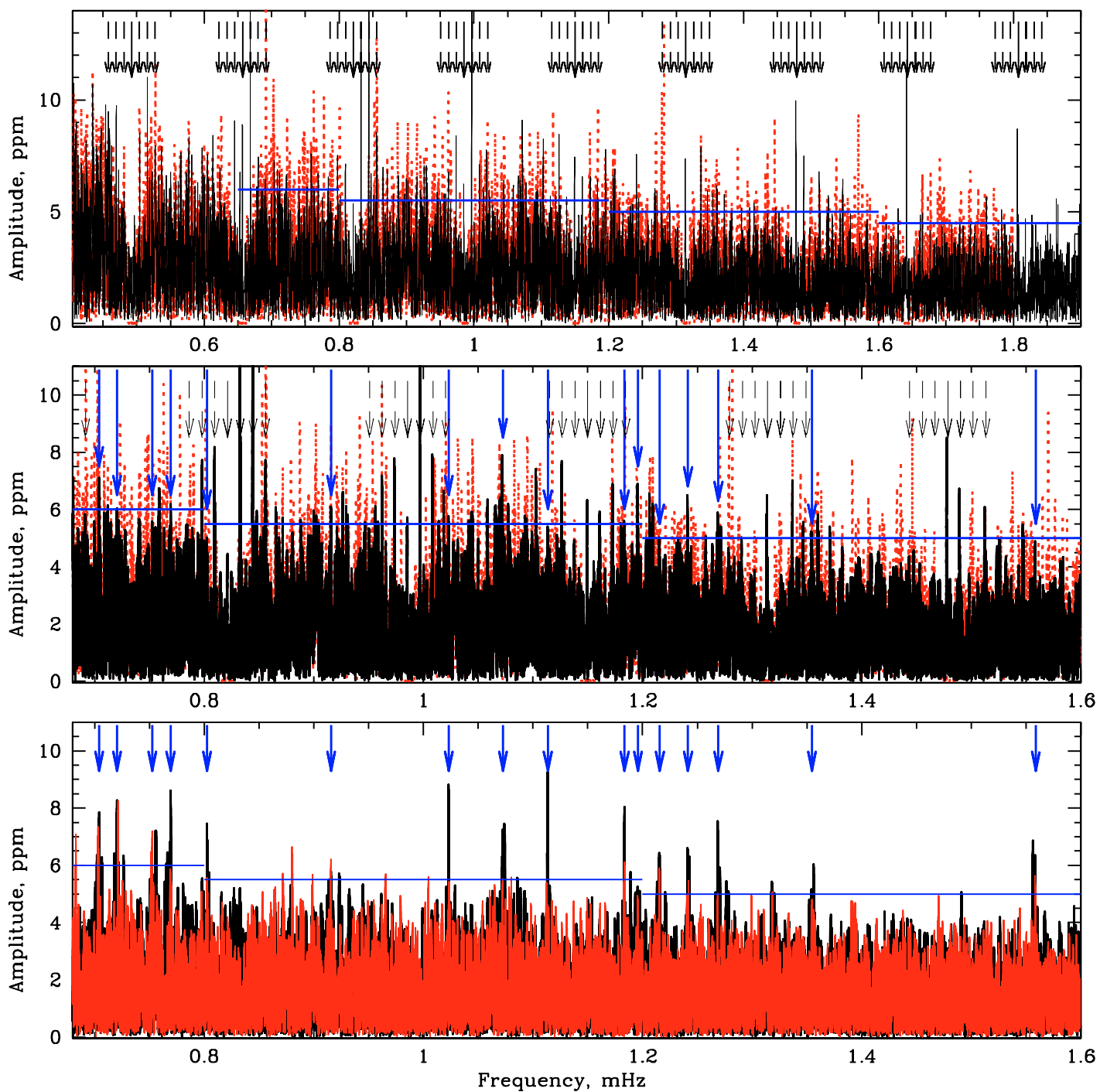

Fig. 1. The upper section shows the new spectrum (black lines) derived from the stand-alone 2004 data and the previously published (red lines) spectrum derived from the same data. The orbital harmonics and their $n * 1 / d$ sidelobes are marked by vertical solid-line and dashed-line arrows, respectively. Middle section: the Fourier amplitude spectrum calculated from the combined 2004-2005 data set processed with the alternative algorithm (black lines), compared to the published (Matthews et al. 2004: red lines) spectrum as a reference. Blue arrows mark the identified p-modes. Bottom section: an artificial spectrum calculated from the shuffled MOST 2004+2005 data with added stochastically-excited sinusoidal signals. Their frequencies are taken from Table 1 . For simplicity, all signals are assigned equal amplitudes $a=10 \mathrm{ppm}$ and lifetimes $L=7$ days (red lines), and $a=15 \mathrm{ppm}, L=3$ days (black lines). Blue arrows mark the identified p-modes. In all sections, the horizontal blue lines denote the $3 \sigma$ detection limits.

providing $s \approx 375 \mathrm{ppm}$, which only moderately exceeds the expected level of $s_{\text {in }}=250 \mathrm{ppm}$. Predictably, the lower noise level brings down average amplitudes in the frequency domain: $\bar{a}(f)=1.4 \pm 1.6 \mathrm{ppm}$ in the $f=1.05-1.25 \mathrm{mHz}$ range, twice lower than in the data from 2004. On the other hand, the standard approach (Guenther et al. 2007), relying uniquely on the SDS2 channel, provides a noise level comparable to the 2004 set.

During the processing of the 2004 data we kept the timebinning approach similar to the original scheme (Matthews et al. 2004); this provided a valuable point-to-point comparison between the two different approaches and retained 214279 flux measurements. For the 2005 data we slightly changed the approach, by time-binning the SDS1 observations centered on the less frequent SDS2 measurements. On average, taking into account the higher rejection rates, the SDS1 data were $\sim 5.5$ times more abundant in the 2005 set. This time-binning approach resulted in 49910 flux measurements.

\section{Results}

Our first step is to compare the frequency spectra calculated by the two data-processing algorithms. As anticipated, the alternative approach, while providing a frequency spectrum of an overall similar appearance, reduces the level of instrumental noise across the spectrum, save a few rare exceptions related to the harmonics of the orbital period (black arrows in Fig. 1).

The next step aims at an optimal combination of the data. A host of potential problems stems from: (a) the different durations of the 2004 and 2005 runs; (b) different planning of the experiments resulting in different levels of stray light; (c) presumably unstable nature of the signals. In the time domain we experimented with different lengths of segments derived from the original time series, varying them from 3 days to 2 weeks and producing a weighted combination of the frequency spectra derived from the segments. This allows us to see that the typical lifetime of signals does not exceed $\sim 1$ week. However, the 


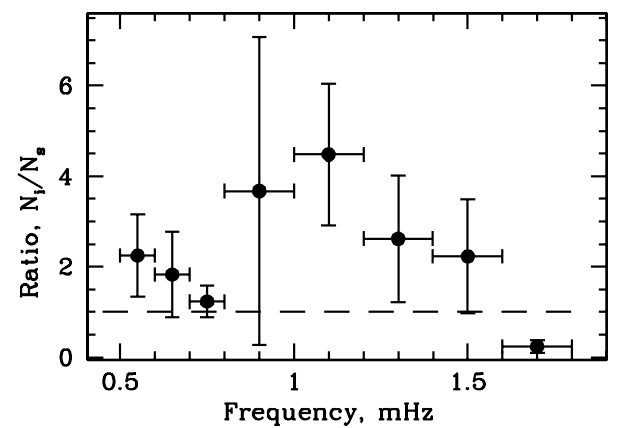

Fig. 2. The numbers $\left(N_{\mathrm{i}}\right)$ of significant (at $3 \sigma$ level) peaks in the $2004+2005$ AS normalized by the corresponding numbers $\left(N_{\mathrm{s}}\right)$ derived from artificial data sets. The vertical bars show the $\pm 1 \sigma$ errors of the ratios. The horizontal dashed line provides a reference level of 1 (the case when all peaks are generated by noise).

segment-based approach comes at a high price, raising, in the region of interest, $f=0.5-2.0 \mathrm{mHz}$, the detectability limits to an unacceptably high level, $a>10 \mathrm{ppm}$. Hence, we finally relied on the simplest and most straightforward approach, by combining the unweighted 2004 and 2005 data into a single set (Fig. 1). Obviously, this results in a further improvement of sensitivity: considering the 3 -sigma level counted from the average amplitude of the noise-dominated signal, one is able to detect coherent oscillations with $a \geq 6.0 \mathrm{ppm}(f=0.7-0.8 \mathrm{mHz}), a \geq 5.5$ $\operatorname{ppm}(f=0.8-1.2 \mathrm{mHz}), a \geq 5.0 \mathrm{ppm}(f=1.2-1.6 \mathrm{mHz})$, $a \geq 4.5 \mathrm{ppm}(f>1.6 \mathrm{mHz})$, to be compared to $a \geq 7.0 \mathrm{ppm}$ ( $f=0.5-2.0 \mathrm{mHz}$ : Matthews et al. 2004) and $a \geq 10-11 \mathrm{ppm}$ (Guenther et al. 2007). Beyond the overall improvement of sensitivity, such a straightforward combination of the 2004 and 2005 data should substantially increase the chance of a positive detection which depends on the presumably short lifetimes of the coherent signals (p-modes) in Procyon A (Leccia et al. 2007) and the stochastic nature of the mode-excitation events (Kjeldsen \& Bedding 1995).

As a first test for the presence of any periodic variations, we derived a Fourier amplitude spectrum (AS) of the combined $2004+2005$ data in the $f=0.5-2.0 \mathrm{mHz}$ domain specifically targeted in numerous observing campaigns. Then we calculated the number of peaks exceeding the $3 \sigma$ detectability levels (see above). We also produced 10 samples via random shuffling of the real data and calculated the incidence of significant signals in the artificial ASa. We normalize the real data by the outcomes of simulations and show them in Fig. 2. There is a surplus of $3 \sigma$ detections around $f \sim 0.8-1.4 \mathrm{mHz}$, which closely matches the spread and location of the power excess determined from numerous independent spectroscopic runs (see Leccia et al. 2007, and references therein).

Further testing brings even more encouraging results. We select a prominent feature at $f=1.072 \mathrm{mHz}$ (Table 1 and Fig. 1) and search for a comb-like pattern (Kjeldsen et al. 1995) in the immediate vicinity of the peak. Scanning the adjacent region with $\delta f=0.0001 \mathrm{mHz}$ steps, we find a maximum response at $f=1.0726 \mathrm{mHz}$ (Fig. 3), related to the large frequency separation of $\Delta f=0.0540 \pm 0.0001 \mathrm{mHz}$, in good agreement with $\Delta f=0.0536-0.0559 \mathrm{mHz}$ consistently provided by all previous spectroscopic campaigns (Eggenberger et al. 2004; Martić et al. 2004; Leccia et al. 2007). The clear presence of a regularlyspaced signal in the MOST-2004 data was noted on multiple occasions: $\Delta f=0.0545 \mathrm{mHz}$ (Régulo \& Roca Cortés 2005), $\Delta f \sim 0.0548 \mathrm{mHz}$ (Matthews, priv. comm. 2005; see also the detailed simulations by Baudin et al. 2008). Can the regular
Table 1. Detected p-modes.

\begin{tabular}{llllll}
\hline \hline$f[\mathrm{mHz}]$ & \multicolumn{4}{c}{ Amplitude, $\begin{array}{c}a \\
\text { [ppm] }\end{array}$} \\
& $2004+2005$ & 2004 & 2005 & $2004^{1}$ \\
\hline 0.7041 & 7.1 & 7.4 & 4.7 & 8.0 & 3 \\
0.7206 & 6.0 & 6.0 & 5.6 & - & 2,4 \\
0.7525 & $(5.6)$ & 6.2 & 5.3 & $(6.6)$ & 3 \\
$(0.7692)$ & 6.2 & 6.3 & 4.0 & 7.5 & 3 \\
0.8027 & $(5.0)$ & 5.7 & 4.0 & - & 2,3 \\
0.9158 & 6.1 & 6.6 & 5.3 & 9.0 & 2,4 \\
1.0232 & 5.8 & 6.0 & 3.6 & - & $2,3,4$ \\
$(1.0726)$ & 6.2 & 9.2 & 8.1 & 7.6 & 3 \\
1.1136 & 5.4 & 5.7 & 3.9 & - & 4 \\
1.1837 & 5.6 & 6.5 & 2.1 & - & 2,3 \\
1.1959 & 6.9 & 7.8 & 3.5 & $(6.2)$ & 2,4 \\
1.2158 & 5.0 & 5.4 & 4.2 & - & 3 \\
1.2415 & 6.5 & 7.6 & 5.2 & $(5.2)$ & 3 \\
1.2692 & 5.9 & 6.1 & 3.3 & $(5.7)$ & $2,3,4$ \\
1.3547 & 5.2 & 5.9 & 3.3 & $(6.0)$ & 3 \\
1.5590 & 4.9 & 4.9 & 4.4 & - & 2,4 \\
\hline
\end{tabular}

${ }^{1}$ Matthews et al. (2004).

${ }^{2}$ Eggenberger et al. (2004).

${ }^{3}$ Martić et al. (2004) ${ }^{4}$ Leccia et al. (2007); raw data and fits to Ref. 2.

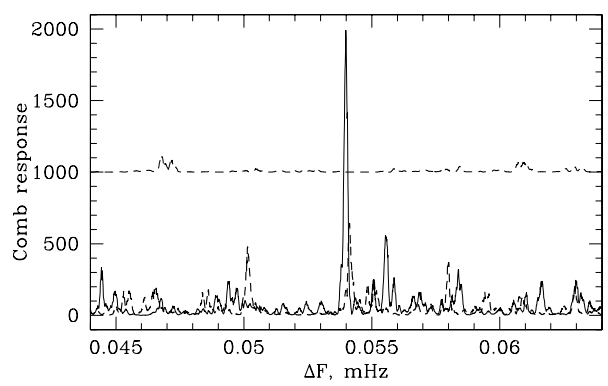

Fig. 3. The comb-response functions obtained for the central frequencies $f=1.0726 \mathrm{mHz}$ (full line), $f=0.9158 \mathrm{mHz}$ (dashed line) and $f=1.6424 \mathrm{mHz}$ (dashed line, offset for clarity). The tallest peak corresponds to a large separation $\Delta f=0.0540 \pm 0.0001 \mathrm{mHz}$, with the error derived from the FWHM of the peak.

spacing of signals be caused by an unaccounted component of the stray light contributing to the $n * v_{\text {orb }} / 3$ orbital harmonics with $0.0548 \mathrm{mHz}$ separation? Probably not, as the newly derived $\Delta f$ is more than sufficiently distanced from the instrumental component. Moreover, we find only a very weak trace of the instrumental signal in the comb-generated response at $f=1.0726 \mathrm{mHz}$ and its complete lack at $f=1.6424 \mathrm{mHz}$ (Fig. 3: note that $f=1.6424 \mathrm{mHz}$ matches an orbital harmonic).

The presence of a regular "comb" of periodic signals around $f=1.072 \mathrm{mHz}$ prompted the final step of the analysis. We compiled a list of frequencies presumably related to $\mathrm{p}$-modes (Eggenberger et al. 2004; Martić et al. 2004; Leccia et al. 2007) and searched for corresponding significant peaks in our 2004+2005 AS. We limited our search to the immediate surroundings of the published frequencies, i.e., to the $\pm \delta f \lesssim$ $0.002 \mathrm{mHz}$ intervals, where $\delta f$ is an uncertainty related to frequency resolution in the AS derived on $\sim 1$ week-long runs. Note that a formal frequency resolution in the combined 2004+2005 AS reaches $3 \times 10^{-5} \mathrm{mHz}$. We recorded the maxima which were very close to, or above, the corresponding $3 \sigma$ levels for the given frequencies. Facing the rapidly rising level of instrumental+intrinsic (granulation?) noise, we disregarded all positive detections with frequencies below $f=0.7 \mathrm{mHz}$. We also eliminated all positive detections (4 cases) which matched high 
harmonics of the orbital period and their $1 / \mathrm{d}$ side lobes. We present the results in Table 1 , where in parentheses we retain some prominent signals despite their relatively large deviations from the published frequencies. As a reference, we provide the amplitudes of signals in the separate subsets from 2004 and 2005 , even if they do not exceed detection limits. We compare our positive $2004+2005$ detections with signals exceeding (or being reasonably close to) the detectability limits in the previously published MOST-2004 data (Matthews et al. 2004), finding $\sim 55 \%$ of positive matches, both in frequency and amplitude. On average, one may expect $2.4 \pm 1.2(\sim 20$ in total) noise-generated signals exceeding the imposed $3 \sigma$ threshold in an $0.1 \mathrm{mHz}$-wide bin placed in the region of interest, $f=0.7-1.6 \mathrm{mHz}$. Hence, the chance for an accidental match between a noise-generated peak and a pre-determined signal registered in the previous spectroscopic campaigns (Eggenberger et al. 2004; Martić et al. 2004) is $\leq 45 \%$, i.e., at least $50 \%$ of the frequencies provided in Table 1 should be geniune. The 16 signals identified as p-modes, plus 20 noise-generated peaks do not account for all positive detections, 46 signals in the $f=0.7-1.6 \mathrm{mHz}$ range. The origin of the 10 remaining peaks is unclear.

The average amplitude of the recovered signals, $a=5.8 \pm$ $0.6 \mathrm{ppm}$, closely matches the $a=7.3 \pm 1.1 \mathrm{ppm}$ level inferred from the ground-based spectroscopy (Leccia et al. 2007) and $a=8.5 \pm 2$ ppm upper limit from WIRE photometry (Bruntt et al. 2005). Such low-amplitude signals were inevitably swamped by instrumental noise in the previous reduction of the stand-alone data from 2004 and 2005. The unusually low level of the detected signals may be related to the anticipated short life-time of the modes, $2.0 \pm 0.4 \mathrm{~d}$ (Leccia et al. 2007). Indeed, one may re-shuffle the original 2004-2005 MOST data and add 16 sinusoidal signals with the frequencies matching the identified p-modes from Table 1. For simplicity, we assign to the signals the same amplitude $a_{\text {art }}$ and lifetime $L$, let the phases of the signals vary randomly, then calculate the resulting ASa and show them in Fig. 1 . In both shown cases, $a_{\text {art }}=10 \mathrm{ppm}, L=7$ days, and $a_{\text {art }}=15 \mathrm{ppm}, L=3$ days, one may detect up to $\sim 80-90 \%$ of the artificial signals. The $a_{\text {art }}=10 \mathrm{ppm}$ case provides the average amplitude $\left\langle a_{\text {art }}\right\rangle=5.0 \pm 0.8 \mathrm{ppm}$, and $a_{\text {art }}=15 \mathrm{ppm}$ case results in $\left\langle a_{\text {art }}\right\rangle=7.1 \pm 1.2 \mathrm{ppm}$ and a slightly better detection rate, thus proving the starting assumption about the short lifetime of the modes. On the other hand, the relative low signal/noise ratio of the data and, presumably, interaction of the stochastically excited modes affects the derived frequencies of artificial signals $\left(f_{\text {art }}\right):\left\langle\left|f_{\text {obs }}-f_{\text {art }}\right|\right\rangle \approx 9 \times 10^{-5} \mathrm{mHz}$, to be compared to the formal frequency resolution $\delta f=3 \times 10^{-5} \mathrm{mHz}$ in the combined 2004+2005 data.

Recently Bedding (2007, priv. comm.; see also Kjeldsen \& Bedding 1995) brought to our attention two effects which may influence the estimate of amplitudes of the detected p-modes. Namely, the [frequency-dependent] instrumental noise may lead to a systematic over-estimate of amplitudes of the real signals. On the other hand, the presumably short life-time of the detected modes (as compared to the length of the MOST observations) produces Lorentz-broadened profiles, thus leading to underestimated amplitudes. These effects can be accurately evaluated (and thus, disentangled) if: (a) the signals are detectable at a high signal-to-noise ratio, $S / N \gg 3$; (b) the instrumental noise can be treated as [practically] frequency-independent in any narrow $(\sim 0.1 \mathrm{mHz})$ frequency interval and, more importantly, such noise can be treated as stationary (time-independent). Neither one applies to the MOST data, hence we provide uncorrected estimates of amplitudes.

To investigate the impact of these effects, we constructed an average, normalized profile from the signals related to the positive detections of p-modes (Table 1) and smoothed it via 2point binning. One may compare this profile with an average profile of noise-related signals, which were selected from the immediate vicinities of the signals related to p-modes. In addition, these noise-related peaks closely match the amplitudes of the mode-related signals. There is a noticeable $\sim 30 \%$ pedestal which is related to the instrumental noise. There is also some, rather marginal, [Lorentz-related?] broadening of the p-mode profile, in accordance with expectations. However, severe blending problems related to low $S / N$ levels make impractical any precise evaluations of the broadening. With these effects working in opposite directions, we conclude that the derived average p-mode amplitude, $a=5.8 \mathrm{ppm}$, may carry a substantial uncertainty, $\pm 1.0-1.5 \mathrm{ppm}$ (i.e. twice the formal, $\pm 0.6 \mathrm{ppm}$, value) and may be considered as an upper limit for mode detection in these data.

Acknowledgements. The author is grateful to the MOST team for making the Procyon data available through the Canadian Astronomy Data Centre (CADC; operated by the Hertzberg Institute of Astrophysics, national Research Council of Canada). Numerous suggestions of the anonymous referee helped to improve presentation of the results.

\section{References}

Aerts, C., Marchenko, S. V., Matthews, J. M., et al. 2006, ApJ, 642, 470 Baudin, F., Appourchaux, T., Boumier, P., et al. 2008, A\&A, 478, 461 Bedding, T. R., \& Kjeldsen, H. 2006, ESA Special Publication, 624, 25 Bedding, T. R., \& Kjeldsen, H. 2007, Comm. in Asteroseismology, in press [arXiv: 0705.1376]

Bedding, T. R., Kjeldsen, H., Bouchy, F., et al. 2005, A\&A, 432, L43

Bruntt, H., Kjeldsen, H., Buzasi, D. L., Bedding, T. R., et al. 2005, ApJ, 633, 440

Guenther, D. B., Kallinger, T., Reegen, P., et al. 2007, Comm. in Asteroseismology, 151, 5

Eggenberger, P., Carrier, F., Bouchy, F., Blecha, A., et al. 2004, A\&A, 422, 247 Kjeldsen, H., \& Bedding, T. R. 1995, A\&A, 293, 87

Kjeldsen, H., Bedding, T. R., Viskum, M., Frandsen, S., et al. 1995, AJ, 109, 1313

Leccia, S., Kjeldsen, H., Bonanno, A., et al. 2007, A\&A, 464, 1059

Martić, M., Schmitt, J., Lebrun, J.-C., et al. 1999, A\&A, 351, 993

Martić, M., Lebrun, J.-C., Appourchaux, T., \& Korzennik, S. G. 2004, A\&A, 418, 295

Matthews, J. M., Kusching, R., Guenther, D. B., et al. 2004, Nature, 430, 51, Erratum: 430, 921

Régulo, C., \& Roca Cortés, T. 2005, A\&A, 444, L5

Walker, G., Matthews, J., Kuschnig, R., et al. 2003, PASP, 115, 1023 